This item was submitted to Loughborough's Research Repository by the author.

Items in Figshare are protected by copyright, with all rights reserved, unless otherwise indicated.

\title{
Classical effective Hamiltonians, Wigner functions, and the sign problem
}

PLEASE CITE THE PUBLISHED VERSION

PUBLISHER

(C) American Physical Society

LICENCE

CC BY-NC-ND 4.0

REPOSITORY RECORD

Samson, J.H.. 2019. "Classical Effective Hamiltonians, Wigner Functions, and the Sign Problem". figshare. https://hdl.handle.net/2134/1311. 


\title{
Classical effective Hamiltonians, Wigner functions, and the sign problem
}

\author{
J. H. Samson* \\ Department of Physics, Loughborough University of Technology, Loughborough, Leics LE11 3TU, England
}

(Received 4 March 1994; revised manuscript received 7 July 1994)

\begin{abstract}
In the functional-integral technique an auxiliary field, coupled to appropriate operators such as spins, linearizes the interaction term in a quantum many-body system. The partition function is then averaged over this time-dependent stochastic field. Quantum Monte Carlo methods evaluate this integral numerically, but suffer from the sign (or phase) problem: the integrand may not be positive definite (or not real). It is shown that, in certain cases that include the many-band Hubbard model and the Heisenberg model, the sign problem is inevitable on fundamental grounds. Here, Monte Carlo simulations generate a distribution of incompatible operators - a Wigner function - from which expectation values and correlation functions are to be calculated; in general no positive-definite distribution of this form exists. The distribution of time-averaged auxiliary fields is the convolution of this operator distribution with a Gaussian of variance proportional to temperature, and is interpreted as a Boltzmann distribution $\exp \left(-\beta V_{\text {eff }}\right)$ in classical configuration space. At high temperatures and large degeneracies this classical effective Hamiltonian $V_{\text {eff }}$ tends to the static approximation as a classical limit. In the low-temperature limit the field distribution becomes a Wigner function, the sign problem occurs, and $V_{\text {eff }}$ is complex. Interpretations of the distributions, and a criterion for their positivity, are discussed. The theory is illustrated by an exact evaluation of the Wigner function for spin $s$ and the effective classical Hamiltonian for the spin- $\frac{1}{2}$ van der Waals model. The field distribution can be negative here, more noticeably if the number of spins is odd.
\end{abstract}

\section{INTRODUCTION}

Functional integration is an important computational tool in areas ranging from the statistical mechanics of correlated electrons to nuclear structure. In studies of magnetism and superconductivity, it is useful to be able to deal directly with the relevant order parameter field (or auxiliary field coupling to it) as the variables of the system. One traces out the electronic degrees of freedom, to leave a functional integral over these variables. Computing power now available allows quantum Monte Carlo evaluation of the functional integrals for model Hamiltonians, giving nonperturbative and (in principle) numerically exact results. ${ }^{1}$

\section{A. The sign problem}

The basis of Monte Carlo integration is the Metropolis algorithm: importance sampling allows integration, over a high-dimensional space, of a function varying by many orders of magnitude. In a classical simulation, points in phase space are typically sampled with probability given by the Boltzmann factor $\exp (-\beta E)$. In a quantum simulation, the corresponding quantity is the weight of a path, which is not necessarily positive (or indeed even real). Nonpositivity is not a formal difficulty in principle; indeed, Feynman has argued that one should not be afraid of using negative probabilities as intermediate results. $^{2}$ In practice, at low temperatures, the integrand may be a rapidly oscillating but sparsely sampled function, rendering numerical averages highly unstable and unreliable. This sign problem (or phase problem) has been a serious impediment to Monte Carlo calculations. First noted in fermion calculations, ${ }^{3}$ it also appears, for example, in spin and Jahn-Teller ${ }^{4}$ systems. The current work does not attempt to overcome the problem; rather, it identifies a class of situations in which the sign problem appears to be inevitable on fundamental grounds, and its neglect may hide relevant physics. (In a similar way, neglect of the related topological terms in onedimensional Heisenberg models would discard spin quantization and lose distinctions between integer and half-integer spins.) For example, in an isotropic ferromagnetic one is interested in the order parameter. We shall show that common quantum Monte Carlo techniques are attempts to generate a probability distribution - a Wigner function - for all components of the total spin. Such distributions cannot in general be positive definite.

\section{B. Classical effective Hamiltonians}

Classical and quantum formulations of statistical mechanics differ in structure. Nevertheless, at finite temperatures the results should be qualitatively in agreement for the following reason. ${ }^{5}$ A quantum system in $d$ dimensions can be mapped onto a classical system in $d+1$ dimensions, with width $\beta=1 / k T$ in the imaginary time direction, as exploited in functional integration. The critical behavior crosses over to a classical $d$-dimensional fixed point once the correlation length becomes comparable with this width. There are subtleties in the application of this simple argument to the auxiliary-field functional integral, as the resulting fermion determinant is a highly nonlocal functional of the field. However, if the 
interactions are exponentially damped at large distances (as might be expected at finite temperature), and an auxiliary field of the correct symmetry is used, one might expect a finite-temperature quantum phase transition to be in the same universality class as the corresponding transition in a classical system. This motivates modeling of quantum statistical mechanics at finite temperature by a classical effective Hamiltonian $V_{\text {eff }}$ in $d$ dimensions, obtained formally by a renormalization-group transformation in which all nonzero frequency modes in the time direction are integrated out.

Such attempts to map quantum onto classical statistical mechanics date from the first decade of quantum mechanics. ${ }^{6}$ The approach that will be developed here is to find the distribution in classical phase space or configuration space and interpret it as a Boltzmann factor $\exp \left(-\beta V_{\text {eff }}\right)$ over the classical space. For example, the partition function of a particle of mass $m$ in a potential $V(x)$ is given by the path integral ${ }^{7}$

$$
Z=\int \mathscr{D} x(\tau) \exp \int_{0}^{\beta}\left[-\frac{m}{2 \hbar^{2}}\left[\frac{d x}{d \tau}\right]^{2}-V(x(\tau))\right] d \tau
$$

The paths are classified according to the mean position of the particle on the path,

$$
\bar{x}=k T \int_{0}^{\beta} x(\tau) d \tau .
$$

The integral is evaluated over all nonzero frequency fluctuations about the mean position, leaving an ordinary integral over $\bar{x}$ :

$$
Z=\int_{-\infty}^{\infty} d \bar{x} \exp \left[-\beta V_{\text {eff }}(\bar{x})\right]
$$

The effective Hamiltonian $V_{\text {eff }}$ is essentially a smeared version of the original potential: the particle explores a neighborhood of range increasing with inverse temperature. (The term effective Hamiltonian, rather than effective potential, is used here for $V_{\text {eff }}$ to emphasize that there is no dynamics. The kinetic energy has already been absorbed into the temperature dependence of the Hamiltonian.) The Feynman-Jensen inequality $F \leq F_{0}+\left\langle S-S_{0}\right\rangle$ allows variational approximations to $V_{\text {eff }}$, if the integrand in Eq. (1.1) is positive, which is the case for spinless particles in time-reversal symmetric fields. ${ }^{8,9}$ (Alternative approaches are based on expansions of the effective Hamiltonian. ${ }^{10}$ ) This Hamiltonian can then be used in a classical Monte Carlo simulation of a many-body system. Such methods have been successfully applied to anharmonic potentials and, through path integration in phase space rather than configuration space, to easy-plane ferromagnets. ${ }^{11}$ At this point the sign problem arises and other, nonvariational, methods are required, which do not extend to isotropic spin models. Knowledge of the corresponding Hamiltonian for isotropic spins would be of interest; it would, however, need to have an unusual form if it is, for example, to recover the subtle topological effects seen in low-dimensional Heisenberg antiferromagnets. The work presented here therefore addresses the question of this effective Hamiltonian; we shall construct it explicitly for simple cases and give a general formal expression. It tends to smooth classical Hamiltonian at high temperatures but has a singular low-temperature limit: the sign problem implies that it is complex.

\section{Wigner functions}

To develop these ideas, we need to clarify what the weights in a quantum Monte Carlo simulation are probabilities of. The answer is clear enough in a classical simulation. For example, in a nonrelativistic plasma one might use a Maxwell-Boltzmann distribution for the charge density to obtain correlation functions. Alternatively, one could replace the Coulomb interaction by an electrostatic potential - an auxiliary field - and consider the distribution of this potential. This may gain one little in the classical case (although it is useful in transforming spin models into field theories ${ }^{12}$ ).

If we try to describe a quantum system in a classical phase space, we immediately run into the question of the meaning of a joint probability density of incompatible observables. The Wigner function is a distribution of this type, which gives the same expectation values as the density matrix and has other useful properties. ${ }^{13}$ It is not a probability density, as it cannot in general be positive definite. The Wigner function appearing in this paper is $p(\mathbf{S})$, the joint distribution of the components of a spin. ${ }^{14}$

In quantum simulations the variables are time dependent. We shall, however, concentrate on the distribution of time-averaged variables, defined by analogy with Eq. (1.2). Within a quantum simulation, this distribution might be constrained averaging over paths. If the operators are conserved (such as the total spin in an isotropic Hamiltonian), we will show that their distribution defined in this way is a Wigner function. Usually (as in the Hubbard model) it is difficult to work directly with the spin and charge distributions. One might therefore introduce a time-dependent auxiliary-field $\mathbf{u}(\tau)$ coupling to spin, charge, or both, in analogy to the potential in the classical plasma, and work with the distribution of the timeaveraged auxiliary fields. This distribution will turn out to be a Gaussian convolution of the operator distribution, and will therefore be nonpositive at low temperatures. This is our interpretation of the sign problem. It will be interpreted here as a Boltzmann distribution $Z^{-1} \exp \left[-\beta V_{\text {eff }}(\overline{\mathbf{u}})\right]$ in a (not necessarily real) effective Hamiltonian $V_{\text {eff }}$. In both high-temperature and highdegeneracy limits, $V_{\text {eff }}$ tends to the static approximation, a well-known approximation in studies of itinerant magnets.

\section{Outline of paper}

The aim of this paper is thus to make explicit the links between quantum and classical simulations, between the spin and auxiliary field distributions, and between the sign problem and the nonpositivity of the Wigner function. Since a number of ideas are to be related, the next section reviews the coherent-state and auxiliary-field functional integrals and the class of Hamiltonians for 
which nonpositive Wigner functions emerge from the simulation. This class includes the Heisenberg and many-band Hubbard models. These are systems where vector rather than scalar auxiliary fields are required. Although not currently studied by auxiliary-field quantum Monte Carlo methods, they represent the generic case.

Integrating out the finite-frequency modes gives related classical distributions for the time-averaged spins and auxiliary fields, derived in Sec. III. We concentrate on systems with conserved order parameters with noncommuting components, such as the Heisenberg ferromagnet. In such cases, the operator distribution is a Wigner function, and the field distribution tends to this as $T \rightarrow 0$. This demonstrates the inevitability of the sign problem on fundamental grounds. A formal procedure for determining this distribution is proposed. It is often difficult to determine if a distribution is positive definite, and a test based on Bochner's theorem is proposed that does not require explicit evaluation of the distribution. The onionskin structure of the Wigner function $P\left(S_{x}, S_{y}, S_{z}\right)$ for spin $s$ is also exhibited and interpreted. Section IV illustrates the formalism by an exact calculation of the effective Hamiltonian and Wigner function for a van der Waals spin model (an infinite-range Heisenberg model). The final section discusses possible relevance to present and future Monte Carlo calculations.

\section{FORMULATION}

\section{A. Hamiltonian}

We shall be concerned with spin Hamiltonians, such as the Heisenberg model,

$$
H=-\mathbf{B} \cdot \sum_{i} \mathbf{S}_{i}-J_{i j} \mathbf{S}_{i} \cdot \mathbf{S}_{j},
$$

and fermion Hamiltonians, such as the one-band Hubbard model

$$
H=-t_{i j} c_{i s}^{\dagger} c_{j s}+I n_{i \uparrow} n_{i \downarrow} \text {. }
$$

(Repeated indices are to be summed over unless otherwise stated.) The general form of Hamiltonian under consideration is

$$
H=-K_{\mu} \hat{A}_{\mu}-J_{\mu \nu} \hat{A}_{\mu} \hat{A}_{\nu},
$$

where $\left\{\hat{A}_{\mu}\right\}$ are single-particle operators, forming a closed $N$-dimensional algebra, and $\left\{K_{\mu}\right\}$ and $\left\{J_{\mu \nu}\right\}$ are such that $H$ is Hermitian. In the Heisenberg model, these operators are spin components $\left\{S_{i x}, S_{i y}, S_{i z}\right\}$, with $K$ the magnetic field and $J$ the exchange interaction. In the one-band Hubbard model the operators could be particle-hole operators $\left\{c_{i s}^{\dagger} c_{j t}\right\}$, where $i$ labels a Wannier orbital and $s$ the spin eigenvalue, $K$ are hopping matrix elements, and $J$, in this case a very sparse matrix, is the on-site repulsion $I$. The Anderson and Kondo Hamiltonians are also of this form.

\section{B. Functional integration}

At this point we need to review two functional-integral methods, coherent-state and auxiliary-field integration, that form the basis of the rest of the paper. The first stage in functional integration is to divide the imaginarytime interval $0 \leq \tau \leq \beta$ into a large number $L$ of time slices of width $\Delta \tau=\beta / L$ :

$$
\exp (-\beta H)=\mathcal{T} \prod_{l=1}^{L} \exp \left(-\Delta \tau H_{l \Delta \tau}\right)
$$

Here the subscript on $H_{\tau}$ levels the time slice to allow time ordering; the time-ordering symbol $\mathcal{T}$ orders factors $H_{\tau}$ with imaginary time $\tau$ increasing from right to left. Then suitable variables are introduced between each time slice, leading to the coherent-state and auxiliary-field functional-integral methods.

The coherent state functional integral is used principally in studies of spin models. ${ }^{15}$ As certain features are relevant to the present work, the formalism is briefly sketched here. A resolution of the identity, in the form of an overcomplete set of coherent states $\int|\mathbf{n}(\tau)\rangle\langle\mathbf{n}(\tau)| d \mu(\mathbf{n}(\tau))$, is introduced between each time slice. The partition function becomes an integral over a product of matrix elements of the form $\left\langle\mathbf{n}(\tau+\Delta \tau)\left|\exp \left(-\Delta \tau H_{\tau}\right)\right| \mathbf{n}(\tau)\right\rangle$, which can be written as

$$
Z=\int \mathscr{D} \mathbf{n} \exp (-S[\mathbf{n}]) \text {. }
$$

For a spin $s$ the coherent state $|\mathbf{n}\rangle=\left|\left\{\mathbf{n}_{i}\right\}\right\rangle$ corresponds to spins $\mathbf{S}_{i}$ "pointing in the $\mathbf{n}_{i}$ direction": $\mathbf{n}_{i} \cdot \mathbf{S}_{i}\left|\mathbf{n}_{i}\right\rangle=s\left|\mathbf{n}_{i}\right\rangle$. Thus each $\mathbf{n}_{i}(\tau)$ moves on a unit sphere. The action of the spin-s Heisenberg model (2.1) is

$$
\begin{aligned}
S\left[\left\{\mathbf{n}_{i}\right\}\right]= & -i s \sum_{i} S_{\mathrm{WZ}}\left[\mathbf{n}_{i}\right] \\
& -\int_{0}^{\beta}\left[s \mathbf{B} \cdot \sum_{i} \mathbf{n}_{i}(\tau)+s^{2} J_{i j} \mathbf{n}_{i}(\tau) \cdot \mathbf{n}_{j}(\tau)\right] d \tau .
\end{aligned}
$$

The Wess-Zumino or Berry phase term $S_{\mathrm{wz}}$ is the solid angle swept out by the spin in its motion. Spin quantization arises from interference between such terms, also responsible for topological effects in low-dimensional magnets. ${ }^{16}$

Similar coherent states can be defined for arbitrary Lie algebras of the operators $\hat{A}_{\mu},{ }^{17}$ and it is in principle possible to apply the same method to fermion systems. However, the large dimensionality of the (highly overcomplete) space of coherent states makes this less than convenient. The method of choice is the auxiliary-field functional integral, in which a Hubbard-Stratonovich transformation linearizes the Hamiltonian in each time slice. The auxiliary-field method has been used for the Hubbard $^{18}$ and Heisenberg ${ }^{19}$ models and is the basis of the grand canonical and projector Monte Carlo methods. A time-dependent auxiliary field $\mathbf{u}(\tau)=\left\{u_{\mu}(\tau)\right\}$ coupled to the operators $\widehat{\mathbf{A}}$ replaces the interaction term; the partition function of a time-dependent free-particle Hamiltonian $h(\tau)$ is then integrated over time dependences of the field

$$
Z=\frac{\int \prod_{\mu} D u_{\mu}(\tau) \exp \left(-\beta V_{0}[\mathbf{u}]\right) z[\mathbf{u}]}{\int \prod_{\mu} D u_{\mu}(\tau) \exp \left(-\beta V_{0}[\mathbf{u}]\right)}
$$


where

$$
\beta V_{0}[\mathbf{u}]=\int_{0}^{\beta} J_{\mu \nu} u_{\mu}(\tau) u_{v}(\tau) d \tau
$$

and

$$
z[\mathbf{u}]=\operatorname{Tr} \mathcal{T} \exp \left(-\int_{0}^{\beta} h(\tau) d \tau\right),
$$

where

$$
h(\tau)=\left[-K_{\mu}-2 J_{\mu \nu} u_{\nu}(\tau)\right] \hat{A}_{\mu \tau} .
$$

The notation $h(\tau)$ indicates that $h$ has explicit $\tau$ dependence; the subscript $\tau$ in $\hat{A}_{\mu \tau}$ is a label for time-ordering purposes. Different linear combinations of the fields have been used elsewhere; the present scale is chosen here for the resulting agreement between operator and field correlation functions: ${ }^{20}$

$$
\begin{aligned}
& \left\langle\hat{A}_{\mu}(\tau)\right\rangle=\left\langle u_{\mu}(\tau)\right\rangle \\
& \left\langle\mathcal{T} \hat{A}_{\mu}\left(\tau_{1}\right) \hat{A}_{v}\left(\tau_{2}\right)\right\rangle=\left\langle u_{\mu}\left(\tau_{1}\right) u_{\nu}\left(\tau_{2}\right)\right\rangle-\frac{1}{2} J_{\mu \nu}^{-1} \delta\left(\tau_{1}-\tau_{2}\right)
\end{aligned}
$$

and so on, all higher operator and field cumulants being equal. Response functions can therefore be read off from the auxiliary-field correlations. For simplicity, the above equations refer to a positive-definite matrix $J_{\mu \nu}$, corresponding to purely attractive interactions; the Heisenberg model (both ferromagnetic and antiferromagnetic) can be written in this way. (In general, the matrix is blockdiagonalized into positive-definite, zero, and negativedefinite subspaces. Fields corresponding to the negativedefinite subspace are replaced by $i u_{\mu}$ in the integrand, and fields corresponding to the zero subspace are omitted from the integration.)

Much work in itinerant magnetism has used the static approximation, in which the functional integral is replaced by an ordinary integral over time-independent fields. The grand potential of noninteracting electrons in a static field defines the Hamiltonian of the auxiliary field.

$$
Z \approx Z_{\mathrm{st}}=\frac{\int \prod_{\mu} d u_{\mu} \exp \left[-\beta V_{\mathrm{st}}(\mathbf{u})\right]}{\int \prod_{\mu} d u_{\mu} \exp \left[-\beta V_{0}(\mathbf{u})\right]},
$$

where

$$
V_{0}(\mathbf{u})=J_{\mu \nu} u_{\mu} u_{\nu}
$$

and

$$
V_{\text {st }}(\mathbf{u})=V_{0}(\mathbf{u})-k T \ln \operatorname{Tr} \exp \left[\beta\left(K_{\mu}+2 J_{\mu \nu} u_{v}\right) \hat{A}_{\mu}\right]
$$

Electronic structure techniques enable computation of this Hamiltonian $V_{\text {st }}$ for a sample of auxiliary-field configurations; the statistical mechanics can then be investigated by, for example, classical Monte Carlo techniques. ${ }^{21}$ The results are difficult to relate to experimental correlation functions, and would be improved by knowledge of the correct classical effective Hamiltonian to which $V_{\text {st }}$ is an approximation. This was one of the motivations of the present work.

There is considerable freedom in the choice of auxiliary field, and this choice is crucial to the arguments in this paper. This is illustrated by the one-band Hubbard model (2.2). Most authors write the interaction in forms such as

$$
n_{i \uparrow} n_{i \downarrow}=\frac{1}{2} n_{i}-2 S_{i z}^{2}=\frac{1}{4} n_{i}^{2}-S_{i z}^{2},
$$

where

$$
n_{i}=\sum_{s=\uparrow}^{\downarrow} c_{i s}^{\dagger} c_{i s} \text { and } \mathbf{S}_{i}=\frac{1}{2} \sum_{s t} c_{i s}^{\dagger} \sigma_{s t} c_{i t} .
$$

(There is no summation over $i$ here.) This allows use of scalar or Ising ${ }^{22}$ auxiliary fields, coupling to the $z$ component of spin (or to the electron density). The HubbardStratonovich transformation, applied to the first form in Eq. (2.16), gives us the free-particle Hamiltonian

$$
h(\tau)=-t_{i j} c_{i s}^{\dagger} c_{j s}-4 I u_{i}(\tau) S_{i z} .
$$

An alternative is to write the interaction in the manifestly isotropic form

$$
n_{i \uparrow} n_{i \downarrow}=\frac{1}{2} n_{i}-\frac{2}{3} \mathbf{S}_{i}^{2}=\frac{1}{4} n_{i}^{2}-\frac{1}{3} \mathbf{S}_{i}^{2},
$$

which requires a vector auxiliary field, coupling to the total spin, for example,

$$
h(\tau)=-t_{i j} c_{i s}^{\dagger} c_{j s}-\frac{4}{3} I \mathbf{u}_{i}(\tau) \cdot \mathbf{S}_{i}
$$

In the one-band model, both the scalar- and vector-field functional integrals are exact and preserve the SU(2) symmetry (until approximations are made), despite apparent uniaxial anisotropy in the former case. This follows from the isotropy of the interaction term $n_{i \uparrow} n_{i \downarrow}$, which only holds for a single band. In a many-band Hubbard model with interorbital repulsion, the interaction must be explicitly isotropic, as in the right-hand side of Eq. (2.19). The functional integral then requires a vector auxiliary field, acting as a time-dependent magnetic field; the same requirement applies to the Heisenberg model. To make the definitions more precise, suppose that a subset of the operators $\widehat{A}_{\mu}$ appears in the interaction term. If these operators commute at equal time, such as the $S_{i z}$, and $n_{i}$ in the one-band Hubbard model, we talk of a scalar field; otherwise, we talk of a vector field. We shall consider the auxiliary-field method in general, regardless of whether (as in the Heisenberg model) far more efficient Monte Carlo methods exist.

The results in this paper refer to the generic case of a vector auxiliary field. Such considerations do not arise in most current Monte Carlo simulations, for which the Hamiltonians allow a scalar auxiliary field. For example, three-band Hubbard models of cuprate superconductors without interband interactions ${ }^{23}$ are, in this context, oneband models and admit a scalar field. In a more general situation, the $J_{\mu \nu}$ might model configuration interactions in a many-electron atom. If there are $k$ operators on each site, the auxiliary field will be a $k$-component field, with operators commuting only on different sites. It is likely that quantum Monte Carlo methods will be applied 
to such systems in the future, and this paper will consider the consequences.

\section{The quantum Monte Carlo method and the sign problem}

These functional integrals may be computed directly by quantum Monte Carlo techniques. ${ }^{1}$ The Metropolis algorithm performs high-dimensional integration by importance sampling:

$$
\langle f\rangle=\frac{\int w(\mathbf{x}) f(\mathbf{x}) d^{N} \mathbf{x}}{\int w(\mathbf{x}) d^{N} \mathbf{x}}=\lim _{M \rightarrow \infty} \frac{1}{M} \sum_{m=1}^{M} f\left(\mathbf{x}^{(m)}\right),
$$

where the points $\left\{\mathbf{x}^{(m)}\right\}$ in the summation are understood to be selected by random sampling from the weight $w(\mathbf{x})$. In a typical classical simulation this weight is the Boltzmann factor $\exp [-\beta E(\mathbf{x})]$, which is manifestly positive. In a quantum simulation, a natural choice might be to take the integrand from the numerator of Eq. (2.7),

$$
w[\mathbf{u}]=\exp \left(-\beta V_{0}[\mathbf{u}]\right) z[\mathbf{u}],
$$

as the weight. Unfortunately, this is not necessarily positive. Indeed, if the interaction term $u_{\mu} \hat{A}_{\mu}$ violates timereversal invariance (as it does for a magnetic-field coupling to a three-component spin), the weight is complex. In practice the configurations are sampled according to the weight $|w(\mathbf{x})|$, and the sign (or phase factor) $s(\mathbf{x})=w(\mathbf{x}) /|w(\mathbf{x})|$ would then be included in the quantity to be averaged:

$$
\langle f\rangle=\lim _{M \rightarrow \infty} \frac{\sum_{m=1}^{M} f\left(\mathbf{x}^{(m)}\right) s\left(\mathbf{x}^{(m)}\right)}{\sum_{m=1}^{M} s\left(\mathbf{x}^{(m)}\right)} .
$$

Problems arise at low temperatures, as the average sign appearing in the denominator typically decreases exponentially with inverse temperature. ${ }^{24}$ This sign problem (or, in the absence of time-reversal symmetry, phase problem) renders numerical calculations highly unstable at low temperatures. As most quantum Monte Carlo simulations deal with scalar fields and real weights there is no phase problem but (except in certain cases ${ }^{25}$ ) may be a sign problem. A phase problem, rather than a sign problem, is the generic case in quantum Monte Carlo simulations, even for scalar interactions if they are repulsive.

The present author has interpreted the phase problem for individual paths in terms of geometrical phases, by relating the coherent-state and auxiliary-field dynamics. $^{26,27}$ For example, consider the Heisenberg model, ${ }^{19}$ where the free-particle Hamiltonian of Eq. (2.10) is

$$
h(\tau)=-\Delta_{i}(\tau) \cdot \mathbf{S}_{i \tau}, \text { with } \Delta_{i}=2 J_{i j} \mathbf{u}_{j}(\tau) .
$$

This corresponds to a time-dependent magnetic field $\Delta$. In a static field the imaginary-time dynamics would be relaxation towards the ground state in that field. These ground states are precisely the coherent states in the directions of the vectors $\Delta_{i}$ and therefore form a complete set of states, represented by a product of unit

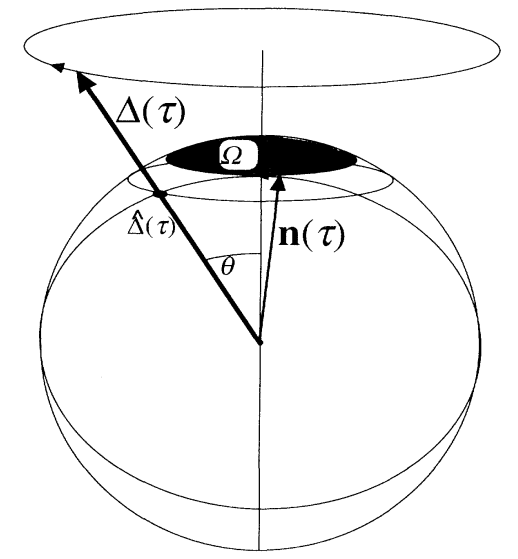

FIG. 1. The relation between the dynamics of the auxiliary field $\Delta$ and of the state $\mathbf{n}$, illustrated for an auxiliary field moving in a cone. The spin relaxes along a great circle towards the instantaneous direction of $\Delta$, sweeping out a narrower cone of solid angle $\Omega$.

spheres and parametrized by the field directions. The time-ordered integral (2.9) is a product of operators $\exp [-h(\tau) \Delta \tau]$ directing a state along a great circle towards the instantaneous ground state. As the field evolves (discontinuously), the state pursues the instantaneous ground state (continuously but not differentiably), as illustrated in Fig. 1. The wave function acquires a geometrical phase factor that depends only on the solid angle $\Omega$ enclosed by the path in state space. The same geometrical phase appears as $S_{\mathrm{wz}}$ in Eq. (2.6). At high temperatures there is insufficient time for the spin to evolve far; it responds to the time-averaged field, recovering the static approximation. At low temperatures, the state can follow a smooth field path almost adiabatically; the phase is then a Berry phase. ${ }^{28}$ There is thus a mapping between field paths in the auxiliary-field integral (2.7) and state paths in the coherent-state integral (2.5). The purpose of the present paper is, however, not to discuss individual paths but statistics of paths. Just as the spin follows the field at low temperatures, and spin cumulants are equal to field cumulants, so will the field distribution tend to the spin distribution, as demonstrated in the following section.

\section{DISTRIBUTIONS}

We are now in a position to investigate the distribution of the time-averaged operators and of the time-averaged auxiliary fields. The former, derived in Sec. III A, will answer questions such as the simultaneous value of noncommuting operators, and will be a Wigner (or related) function. It will not be positive definite, and the sign can be ascribed to the phases in the coherent-state integral.

Section III B relates the auxiliary-field distribution to this Wigner function, implying the sign problem. The distribution is also interpreted as a Boltzmann distribution in a classical (but complex) effective Hamiltonian.

We shall calculate these distributions using the method 
of characteristic functions, ${ }^{29}$ frequently used in the study of Wigner functions. ${ }^{13}$ Suppose $\mathbf{x}$ is a set of random variables; the probability distribution for the $k$-dimensional vector $\mathbf{y}=\mathbf{f}(\mathbf{x})$ is

$$
p(\mathbf{y})=\langle\delta(\mathbf{y}-\mathbf{f}(\mathbf{x}))\rangle .
$$

The exponential form for the $\delta$ function allows us to rewrite this distribution as

$$
p(\mathbf{y})=(2 \pi)^{-k} \int e^{i \lambda \cdot \mathbf{y}} \chi(\lambda) d^{k} \lambda
$$

where the characteristic function $\chi$, the Fourier transform of the distribution, is

$$
\chi(\lambda)=\left\langle e^{-i \lambda \cdot \mathbf{f}(\mathbf{x})}\right\rangle .
$$

It is a generating function for the moments of $y$,

$$
\chi(\lambda)=1-i \lambda_{\mu}\left\langle y_{\mu}\right\rangle-\frac{1}{2} \lambda_{\mu} \lambda_{v}\left\langle y_{\mu} y_{v}\right\rangle+\cdots
$$

and its logarithm generates the connected correlation functions. Although it is rather unconventional to interpret this property of a generating function as arising from a distribution of operators, it will be useful in the current work.

\section{A. Distributions of the operators}

Suppose the components of $\mathbf{y}$ are operators $\widehat{\mathbf{A}}$, a $k$ dimensional subalgebra of the operators $\left\{\hat{A}_{\mu}\right\}$. For example, $\widehat{\mathbf{A}}$ could be the order parameter $\Sigma S_{i}$ in a Heisenberg or Hubbard ferromagnet (so that $k=3$ ). It is still possible to find a distribution that obeys most of the properties of a probability density (apart from positivity). However, operator-ordering ambiguities mean that this distribution is not unique. We define two characteristic functions. Let us first consider the function

$$
\begin{aligned}
\bar{\chi}(\lambda) & =\frac{\operatorname{Tr} \exp (-\beta H-i \lambda \cdot \widehat{\mathbf{A}})}{\operatorname{Tr} \exp (-\beta H)} \\
& =\left\langle\mathcal{T} \exp \left(-i k T \lambda_{\mu} \int_{0}^{\beta} \hat{A}_{\mu}(\tau) d \tau\right]\right\rangle,
\end{aligned}
$$

where

$$
\widehat{O}(\tau)=e^{H \tau} \hat{O} e^{-H \tau}
$$

and

$$
\langle\hat{O}\rangle=\frac{\operatorname{Tr}\left(\hat{O} e^{-\beta H}\right)}{\operatorname{Tr} e^{-\beta H}} .
$$

This is the partition function of the system in an imaginary field $i k T \lambda$ divided by that in zero field, and is also the generating function for static correlation functions,

$$
\bar{\chi}(\lambda)=1-i \lambda_{\mu}\left\langle\hat{A}_{\mu}\right\rangle-\frac{1}{2} \lambda_{\mu} \lambda_{\nu}\left\langle(k T)^{2} \mathcal{T} \int_{0}^{\beta} \int_{0}^{\beta} \hat{A}_{\mu}\left(\tau_{1}\right) \hat{A}_{v}\left(\tau_{2}\right) d \tau_{1} d \tau_{2}\right\rangle+\cdots
$$

We shall interpret its Fourier transform, $\bar{p}(\mathbf{A})$ as the distribution for the time-averaged operator.

This paper concentrates on the conserved case where $\left[H, \hat{A}_{\mu}\right]=0$, satisfied by the ferromagnetic order parameter. In that case Eq. (3.5) simplifies to

$$
\bar{\chi}(\boldsymbol{\lambda})=\chi(\boldsymbol{\lambda}) \equiv\langle\exp (-i \boldsymbol{\lambda} \cdot \hat{\mathbf{A}})\rangle \text {. }
$$

Its Fourier transform

$$
p(\mathbf{A})=(2 \pi)^{-k} \int e^{i \lambda \cdot \mathbf{A}} \chi(\boldsymbol{\lambda}) d^{k} \lambda
$$

is the Wigner function (or $P$ representation in Weylsymmetrized ordering ${ }^{13}$ ) corresponding to the canonical ensemble. The $\mathbf{A}$ in Eq. (3.10) is a vector of real numbers, not of operators. The moments of this distribution are symmetrized correlation functions for equal-time operators as in (3.4). We are interested in whether the distribution $p(\mathbf{A})$ is positive.

At this point we digress to consider the Wigner function of a spin, which does not seem to be widely known but will be of relevance to the distributions. Chandler et al. have derived a Wigner function for a spin $\frac{1}{2}$ in a magnetic field. ${ }^{14}$ We shall generalize their result to arbitrary spin $s$ (but zero field). In that case the characteristic function (3.9) can be expressed in terms of the partition function $Z(B)$ in a magnetic field:

$$
\chi(\lambda)=\frac{Z(i k T \lambda)}{Z(0)}=\frac{1}{2 s+1} \sum_{m=-s}^{s} \exp (-i m \lambda) .
$$

The Fourier transform can be performed: ${ }^{14}$

$$
p(\mathbf{S})=-\frac{1}{2 \pi(2 s+1)} \sum_{m=-s}^{s} \frac{d}{d S} \delta(S-m) .
$$

This Wigner function has an onionlike structure of derivatives of $\delta$ functions on concentric spheres of quantized radius. To check that this rather unfamiliar form is correct, we can integrate over $S_{x}$ and $S_{y}$ to obtain the marginal distribution $p\left(S_{z}\right)$. This vanishes unless the plane of integration is tangent to one of the spheres, in which case the outer (positive) part of the $-\delta^{\prime}$ function is not canceled by the inner part. It is straightforward to perform the integration and obtain the expected result

$$
\begin{aligned}
p\left(S_{z}\right) & =\int_{-\infty}^{\infty} d S_{x} \int_{-\infty}^{\infty} d S_{y} p(\mathbf{S}) \\
& =\frac{1}{(2 s+1)} \sum_{m=-s}^{s} \delta\left(S_{z}-m\right) .
\end{aligned}
$$

This result can be related to the coherent-state functional integral. We show that the distribution of the time-averaged spin

$$
s \overline{\mathbf{n}}=k T \int_{0}^{\beta} s \mathbf{n}(\tau) d \tau,
$$


where the distribution of $\mathbf{n}$ is defined by the coherentstate functional integral (2.5) for a single spin $s$ in zero field, is the Wigner function just calculated. The characteristic function corresponding to $s \overline{\mathbf{n}}$,

$$
\begin{aligned}
\bar{\chi}(\lambda) & =\frac{\int \mathscr{D} \mathbf{n} \exp \left(i s S_{\mathrm{wz}}[\mathbf{n}]-i k T s \lambda \cdot \int_{0}^{\beta} \mathbf{n}(\tau) d \tau\right)}{\int \mathcal{D n} \exp \left(i S_{\mathrm{wz}}[\mathbf{n}]\right)} \\
& =\frac{Z(i k T \lambda)}{Z(\mathbf{0})},
\end{aligned}
$$

is the same characteristic function as that of a spin in (3.11). The Wigner function of a spin is therefore the distribution of the time-averaged path $s \mathbf{n}(\tau)$ in the coherent-state functional integral. This would be obtained by an integration over all paths $\mathbf{n}(\tau)$ constrained to have a time average $\overline{\mathbf{n}}$, as in the definition of the effective Hamiltonian. The same result would appear for other conserved quantities, such as the total magnetization in the Heisenberg model in the absence of a magnetic field.

This is a somewhat paradoxical result: although $|\overline{\mathbf{n}}|<1$ for almost all paths on the sphere, the spin- $\frac{1}{2}$ Wigner function vanishes for $|\overline{\mathbf{n}}| \neq 1$. This suggests that the sign problem is required in order to ensure proper quantization of the appropriate variables, and that attempts to circumvent the sign problem by replacing the weight by its absolute value ${ }^{30}$ may be discarding such physics.

\section{B. Distributions for the auxiliary field}

Practical Monte Carlo calculations use the auxiliaryfield functional integral in preference to the coherent- state functional integral. The dynamics of the states in the latter is tied to that of the fields in the former, as discussed in Sec. II C, and the distribution of the timeaveraged field $\bar{u}_{\mu}=k T \int_{0}^{\beta} u_{\mu}(\tau) d \tau$ will therefore be related to the Wigner functions already discussed. If the functional integral suffers from the sign problem, some of the paths contributing to the field distribution will have negative weight. It will turn out that, even after the integration over nonzero frequency modes, the resulting classical distribution is not positive definite at low temperatures. Indeed, it will tend to the Wigner functions discussed above in the low-temperature limit.

The distribution of the time-averaged field can be interpreted, as in Eq. (1.3), as a Boltzmann distribution of the auxiliary field in a classical effective Hamiltonian $V_{\text {eff }}$,

$$
\begin{aligned}
\bar{P}(\overline{\mathbf{u}}) & =\left\langle\delta\left[\overline{\mathbf{u}}-k T \int_{0}^{\beta} \mathbf{u}(\tau) d \tau\right]\right\rangle \\
& =\frac{1}{Z} \exp \left[-\beta V_{\mathrm{eff}}(\overline{\mathbf{u}})\right],
\end{aligned}
$$

where the expectation values are now taken with respect to the auxiliary-field functional integral. (Upper case $P, X$ will refer to distributions and characteristic functions of the field, while lower case $p, \chi$ refer to functions of the operators.) Equation (3.16) can be considered as a renormalization-group transformation collapsing the time direction to a point. This is in contrast to the static approximation, which represents the first approximation to $V_{\text {eff }}$ and ignores quantum fluctuations.

Insertion in the functional integral (2.7) gives the characteristic function of the distribution $\bar{P}$ as

$$
\begin{aligned}
\bar{X}(\lambda) & =\left\langle\exp \left(-i k T \lambda_{\mu} \int_{0}^{\beta} u_{\mu} d \tau\right]\right\rangle \\
= & \frac{\int \mathscr{D} \mathbf{u} \exp \left(-\beta V_{0}[\mathbf{u}]\right) \operatorname{Tr} \mathcal{T} \exp \left[\int_{0}^{\beta}\left\{\left[K_{\mu}+2 J_{\mu \nu} u_{v}(\tau)\right] \hat{A}_{\mu \tau}-i k T \lambda_{\mu} u_{\mu}(\tau)\right\} d \tau\right)}{\int \mathcal{D u} \exp \left(-\beta V_{0}[\mathbf{u}]\right) \operatorname{Tr} \mathcal{T} \exp \left[\int_{0}^{\beta}\left\{\left[K_{\mu}+2 J_{\mu \nu} u_{\nu}(\tau)\right] \hat{A}_{\mu \tau}\right\} d \tau\right)} .
\end{aligned}
$$

Observing that the coefficient of $\mathbf{u}(\tau)$ in the numerator has become shifted, with respect to that in the denominator, by an amount proportional to $\lambda$, we can immediately complete the square and invert the Hubbard-Stratonovich transformation to obtain

$$
\begin{aligned}
\bar{X}(\lambda) & =\frac{\operatorname{Tr} \exp \left\{\beta\left[K_{\mu} \hat{A}_{\mu}+J_{\mu \nu}\left(\hat{A}_{\mu}-\frac{1}{2} i k T J_{\mu \xi}^{-1} \lambda_{\xi}\right)\left(\hat{A}_{v}-\frac{1}{2} i k T J_{v \pi}^{-1} \lambda_{\pi}\right)\right]\right\}}{\operatorname{Tr} \exp \left(\beta\left[K_{\mu} \hat{A}_{\mu}+J_{\mu \nu} \hat{A}_{\mu} \hat{A}_{v}\right]\right)} \\
& =\exp \left(-\frac{1}{4} k T J_{\mu \nu}^{-1} \lambda_{\mu} \lambda_{v}\right)\left[\frac{\operatorname{Tr} \exp \left(-\beta H-i \lambda_{\mu} \hat{A}_{\mu}\right)}{\operatorname{Tr} \exp (-\beta H)}\right] .
\end{aligned}
$$

The expression in square brackets is the characteristic function $\bar{\chi}(\lambda)$ of Eq. (3.5); the field characteristic function is the operator characteristic function multiplied by a Gaussian factor.

The distribution $\bar{P}$ of the time-average fields is therefore a Gaussian convolution of the distribution $\bar{p}$ of the operators:

$$
\bar{P}(\overline{\mathbf{u}})=\sqrt{\operatorname{det}(\beta J / \pi)} \int d \mathbf{x} \exp \left(-\beta J_{\mu v} x_{\mu} x_{v}\right) \bar{p}(\overline{\mathbf{u}}-\mathbf{x}) .
$$

This is a key result of this paper. In retrospect it should 
not be unexpected, since the operator and auxiliary-field cumulants, apart from the second cumulant, are equal as stated in Sec. II B, but the result does not seem to have been expressed elsewhere in terms of distributions. If the operators in question are conserved, the distribution of the operators is a Wigner function $\bar{p}=p(\mathbf{A})$, and that of the fields coupled to them is a smoothed Wigner function. The variance of the Gaussian is proportional to temperature, so that in the low-temperature limit the field and operator distributions coincide. This is related to the adiabatic following of the field by the spin described in Sec. II C; in addition, the action for the auxiliary fields in the Heisenberg model in the low-temperature limit has a similar form to that of spins. ${ }^{31}$ Because the Wigner function can be negative, the field distribution becomes negative at sufficiently low temperature. This is a manifestation of the sign problem; indeed it demonstrates its inevitablity. Even after averaging over a large number of paths the weight remains negative. The expression (3.20) is, however, not restricted to the case of conserved operators.

The above gives a formal definition of the effective classical Hamiltonian, though not a practical method for its calculation. It is more difficult to calculate the effective Hamiltonian than the partition function; following the above definition (3.5) would entail solving the original interacting Hamiltonian with an additional random spatially varying (but static) imaginary field $i k T \lambda$ and then taking a Fourier transform with respect to the field. Options for approximations are limited by the nonpositivity of the integrand, which precludes standard variational approaches. An expansion parameter is, however, available in the $v$-band Hubbard model. ${ }^{32}$ A $1 / v$ expansion is possible if the operators $\left\{\hat{A}_{\mu}\right\}$ have the form ${ }^{33}$

$$
\widehat{A}_{\mu}=\sum_{a=1}^{v} \widehat{A}_{\mu a},
$$

with

$$
\left[\widehat{A}_{\mu a}, \widehat{A}_{v b}\right]=0 \text { for } a \neq b,
$$

and the $J_{\mu \nu}$ scaling as $1 / v$. In the $v$-band Hubbard model $a$ would be an orbital index. The functional integral then becomes ${ }^{32}$

$$
Z=\frac{\int \prod_{\mu} D u_{\mu}(\tau) \exp \left[v\left[-\beta V_{0}[\mathbf{u}]+\ln z[\mathbf{u}]\right]\right\}}{\int \prod_{\mu} D u_{\mu}(\tau) \exp \left(-v \beta V_{0}[\mathbf{u}]\right)},
$$

where $z[\mathbf{u}]$ is the partition function of a single $(v=1)$ band in the field $\mathbf{u}$. This form suggests a saddle-point expansion about the Hartree-Fock solution; it is, however, better to expand the effective Hamiltonian in powers of $1 / v$ as $^{34}$

$$
V_{\mathrm{eff}}(\overline{\mathbf{u}})=V_{\mathrm{st}}(\overline{\mathbf{u}})+\frac{1}{v} V_{\mathrm{RPA}^{\prime}}(\overline{\mathbf{u}})+\cdots .
$$

The leading term $V_{\text {st }}$, the effective Hamiltonian in the static approximation, already contains all diagrams, with zero-frequency interactions only, and $V_{\mathbf{R P A}^{\prime}}$ is the contribution from quadratic finite-frequency fluctuations of the field, related to the random phase approximation. ${ }^{18}$ Since the effective Hamiltonian is complex at low temperatures, convergence of this expansion may be a delicate matter.

\section{A criterion for positivity}

Since the distributions discussed here involve a highdimensional Fourier transform, it is useful to have a means of checking for positivity that is based on the characteristic function directly. Bochner's theorem ${ }^{29}$ states that a function $\chi(\lambda)$, with $\chi(0)=1$, is the characteristic function of a positive probability density if and only if, for all sets of $n$ points $\left\{\lambda_{i} \in \mathbb{R}^{N}, i=1, \ldots, n\right\}$, and all sets of $n$ complex numbers $a_{i}$, the sum

$$
E \equiv \sum_{i=1}^{n} \sum_{j=1}^{n} a_{i} a_{j}^{*} \chi\left(\lambda_{i}-\lambda_{j}\right) \geq 0 .
$$

This provides a means of showing that a distribution is not positive definite without evaluating a Fourier transform. Faced with a characteristic function, choose a suitable set of points $\lambda_{i}$, for convenience at the vertices of a regular polyhedron. Then minimize $E$ with respect to the $\left\{a_{i}\right\}$, subject to the spherical constraint $\sum\left|a_{i}\right|^{2}=1$. If the minimum is negative, the underlying distribution cannot be positive. (This can be thought of as a tight-binding band-edge calculation.) For fixed $\left\{\lambda_{i}\right\}$, the condition (3.25) is a necessary but not sufficient condition for the distribution to be positive. In a three-dimensional space with a spherically symmetric characteristic function we could take four points $\lambda_{i}$ at the vertices of a regular tetrahedron of side $d$, and all $a_{i}$ to be $\frac{1}{2}$. Then $E=1+3 \chi(d)$, which can be negative in the case of a half-integer spin, since $\chi(2 \pi)=-1$ from Eq. (3.11). For integer spin it is harder, but possible, to find a set of points for which the sum is negative. This agrees with the result, obtained by explicit Fourier transformation, that the Wigner function is not positive.

\section{ILLUSTRATIVE EXAMPLE: THE van der WAALS SPIN MODEL}

The van der Waals spin model comprises $v$ interacting spin- $\frac{1}{2}$ particles with an equal interactions $J / \nu$ between each spin,

$$
H=-\frac{J}{v}\left[\sum_{a=1}^{v} \mathbf{S}_{a}\right]^{2}-\mathbf{B} \cdot \sum_{a=1}^{v} \mathbf{S}_{a} .
$$

(The self-interaction terms $\mathbf{S}_{a} \cdot \mathbf{S}_{a}$ give a shift of $-3 J / 4$ in the energy, which we ignore.) This Hamiltonian is exactly soluble, ${ }^{35}$ and is described here to illustrate some of the features of the effective Hamiltonian. The total spin $\sum \mathbf{S}_{a}$ is an example of an operator of the form (3.21); furthermore, it is conserved in zero field. The partition function is

$$
\begin{aligned}
Z(\mathbf{B})=\sum_{S=0 \text { or } \frac{1}{2}}^{v / 2} & \frac{v !(2 S+1)]}{(v / 2-S) !(v / 2+S+1) !} \\
& \times \frac{\sinh \left[\frac{1}{2} \beta B(2 S+1)\right]}{\sinh \left[\frac{1}{2} \beta B\right]} e^{\beta J S(S+1) / v} .
\end{aligned}
$$


The lower limit of the sum is 0 (or $\frac{1}{2}$ ) if $v$ is even (or odd).

From the above equation, we immediately obtain the characteristic function in zero field as

$$
\begin{aligned}
\chi(\lambda) & =\frac{Z(i k T \lambda)}{Z(0)} \\
= & \frac{\sum_{S=0 \text { or } \frac{1}{2}}^{v / 2}\left[\frac{(2 S+1) e^{\beta J S(S+1) / v}}{(v / 2-S) !(v / 2+S+1) !} \sum_{m=-S}^{S} e^{i \lambda m}\right]}{\sum_{S=0 \text { or } \frac{1}{2}}^{v / 2} \frac{(2 S+1)^{2} e^{\beta J S(S+1) / v}}{(v / 2-S) !(v / 2+S+1) !}} .
\end{aligned}
$$

The Wigner function for the total spin $\mu$ of the van der Waals model is therefore a generalization of the calcula- tion in the previous section for a single spin,

$$
p(\boldsymbol{\mu})=-\frac{1}{2 \pi \mu} \sum_{m=-S}^{S} F(m) \delta^{\prime}(\mu-m),
$$

where $F(m)$ is the probability that $S_{z}=m$ :

$$
F(m)=\frac{\sum_{S=|m|}^{v / 2} \frac{(2 S+1) e^{\beta J S(S+1) / v}}{(v / 2-S) !(v / 2+S+1) !}}{\sum_{S=0 \text { or } \frac{1}{2}}^{v / 2} \frac{(2 S+1)^{2} e^{\beta J S(S+1) / v}}{(v / 2-S) !(v / 2+S+1) !}}
$$

The field distribution and effective Hamiltonian are obtained by smearing out the $\delta^{\prime}$ distributions as in Eq. (3.20):

$$
P(\mathbf{u})=\left[\frac{\beta J}{v \pi}\right]^{3 / 2} e^{-\beta J u^{2} / v} \sum_{m=-v / 2}^{v / 2} F(m)\left[\cosh \frac{2 \beta J u m}{v}-\frac{m}{u} \sinh \frac{2 \beta J u m}{v}\right] e^{-\beta J m^{2} / v}
$$

This distribution is negative for certain ranges of $u$ at low temperatures. The distribution of the total spin in the Heisenberg model (and indeed the Hubbard model) would be of similar form, but with combinatorial factors $F(m)$ that would be impossible to evaluate analytically. The corresponding effective Hamiltonian,

$$
V_{\mathrm{eff}}(\mathbf{u})=-k T \ln [Z P(\mathbf{u})],
$$

has a nonzero imaginary part at points where the distribution is negative. Figure 2 compares this with the corre- sponding effective Hamiltonian (2.15) in the static approximation,

$$
\begin{aligned}
V_{\mathrm{st}}(\mathbf{u})= & -\frac{3 k T}{2} \ln \left[\frac{\beta J}{v \pi}\right)+J u^{2} / v \\
& -v k T \ln [2 \cosh (\beta J u / v)] .
\end{aligned}
$$

These effective Hamiltonians coincide in the hightemperature limit. At low temperatures $V_{\text {eff }}$ has a nonzero imaginary part, while $V_{\text {st }}$ remains real but leads
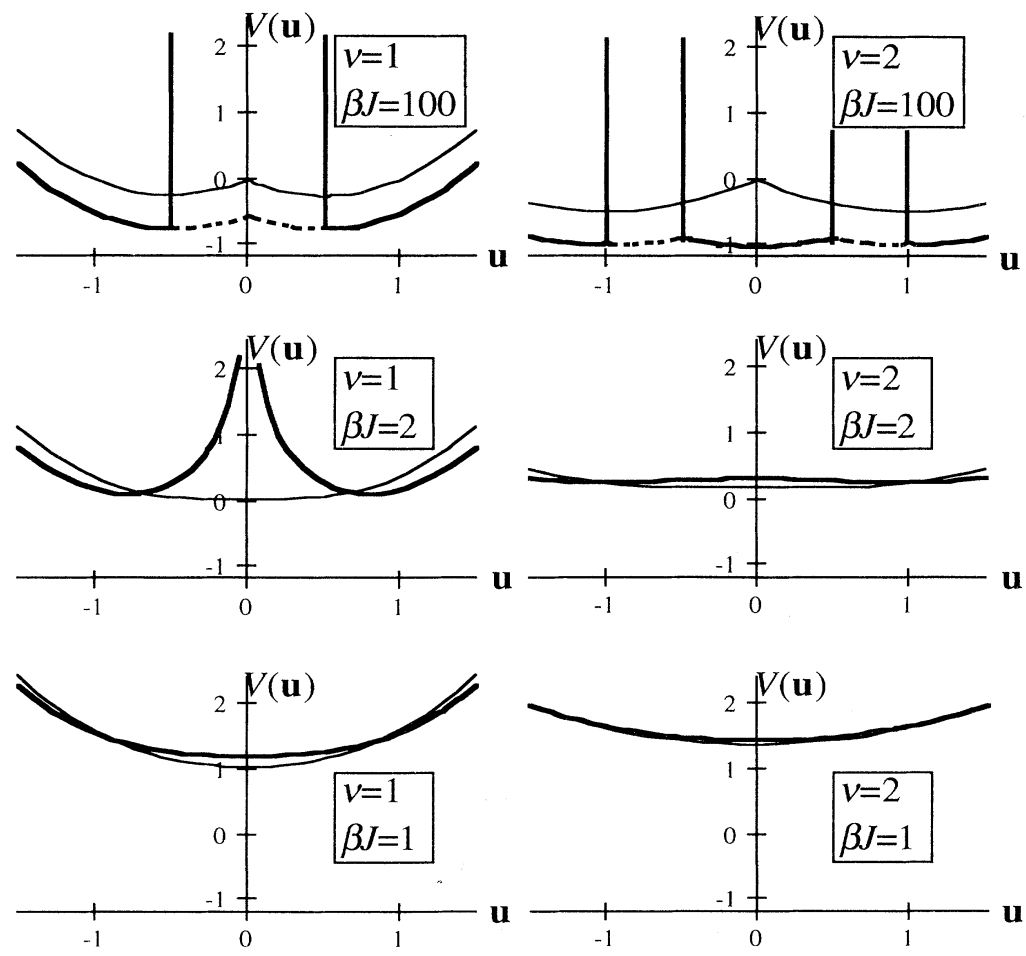

FIG. 2. The real part of the effective Hamiltonian (in units of $J$ ) for the auxiliary field in the van der Waals spin model (bold), and the static approximation to the same Hamiltonian (thin line), for one and two spins at various temperatures. In the dashed regions the weight is negative and the effective Hamiltonian is therefore complex. Vertical lines indicate logarithmic singularities. 
to a negative heat capacity. ${ }^{27,36}$ Integration of the exact effective Hamiltonian of course gives the correct partition function.

\section{DISCUSSION}

This paper has considered the distribution of time averages both of the spins (or similar operators) and of the auxiliary fields appearing in a functional integral. The main result is the identification of the sign problem with the nonpositivity of the Wigner function. Just as the correlation functions of the auxiliary fields are related to the correlation functions of the corresponding operators, the distribution of the time-averaged fields is related to the distribution of the operators. The field distribution is the convolution of the operator distribution with a Gaussian of variance proportional to temperature. This paper has concentrated on the distribution and effective Hamiltonian for conserved quantities. In that case the operator distribution is a Wigner function, which, if the operators do not commute, need not be positive at any temperature - as shown by explicit calculation of a spin Wigner function. The distribution of the time-averaged auxiliary field, as a smoothed form of the operator distribution, becomes the Wigner function in the lowtemperature limit. If an auxiliary field couples to noncommuting conserved operators, even a high-dimensional average of paths can have negative weight. This therefore establishes the inevitability of the sign problem.

This might suggest a rather bleak outlook for a class of quantum Monte Carlo calculations. It is therefore useful to consider what features have led to this conclusion. First, the discussion only refers to the auxiliary-field (or grand canonical) method, and the closely related ground-state projector method. It shows that the sign problem occurs if the operators coupled to the auxiliary field generate a non-Abelian symmetry group; that is, they commute with the Hamiltonian but not with each other. This is necessary if the simulation is to study the total magnetization of an isotropic model, such as the
Heisenberg or Hubbard model.

A further element is the form of the effective classical Hamiltonian. This describes the system as a classical Hamiltonian for the time average of the appropriate quantum variables. It is constructed to give the correct thermodynamics, but is difficult to calculate in general; an expansion has been proposed. The effective classical spin Hamiltonian is necessarily complex as a result of the sign problem. The effective Hamiltonian for auxiliary fields is a smoothed version of that for the original variables, and is therefore less singular; it is also accessible by Monte Carlo simulation.

This says nothing about the sign problem in cases where a scalar auxiliary field couples to only one spin component, which correspond to most current studies. This is allowed for the one-band Hubbard model, but not for the interactions described above. With nonconserved scalar fields the commutator of the operators may vanish at equal time only:

$$
\left[\hat{A}_{\mu}(\tau), \hat{A}_{v}\left(\tau^{\prime}\right)\right] \sim\left(\tau-\tau^{\prime}\right)^{n}
$$

In the vector field cases considered here, $n=0$. For scalar fields the sign problem might arise from the same effects but at a higher order. The resulting distribution of nonconserved observables (for example, long-wavelength modes in a ferromagnetic or staggered magnetization in an antiferromagnet) is left for subsequent work.

This paper has not attempted to circumvent the sign problem, but rather to put it in a broader context and identify situations in which it is inevitable on fundamental grounds. It suggests that neglect of the sign may hide important physics, just as neglect of the related topological terms in one-dimensional Heisenberg models hides the distinction between integer and half-integer spins. Nevertheless, identification of a source of the sign problem would be an aid in seeking auxiliary-field couplings that can avoid it. In essence, the field would have to couple to commuting operators, which is not possible for general on-site interaction with more than two states.
*Electronic address: j.h.samson @ lut.ac.uk

${ }^{1}$ W. von der Linden, Phys. Rep. 220, 53 (1992) gives a recent review.

${ }^{2}$ R. P. Feynman, in Quantum Implications, edited by B. J. Hiley and F. D. Peat (Routledge, London, 1987), p. 235.

${ }^{3}$ R. Blankenbecler, D. J. Scalapino, and R. L. Sugar, Phys. Rev. D 24, 2278 (1981).

${ }^{4}$ P. de Vries, Ph.D. thesis, Universiteit van Amsterdam, 1991.

${ }^{5}$ J. A. Hertz, Phys. Rev. B 14, 1165 (1976).

${ }^{6}$ E. Wigner, Phys. Rev. 40, 749 (1932).

${ }^{7}$ R. P. Feynman and A. R. Hibbs, Quantum Mechanics and Path Integrals (McGraw-Hill, New York, 1965), Chap. 10.

${ }^{8}$ R. P. Feynman and H. Kleinert, Phys. Rev. A 34, 5080 (1986).

${ }^{9}$ R. Giachetti and V. Tognetti, Phys. Rev. Lett. 55, 912 (1985); Phys. Rev. B 33, 7647 (1986).

10J. Cao and G. A. Voth, J. Chem. Phys. 100, 5093 (1994).

${ }^{11}$ A. Cuccoli, V. Tognetti, P. Verrucchi, and R. Vaia, Phys. Rev. A 45, 8418 (1992); B 46, 11601 (1992).

${ }^{12}$ See, e.g., D. J. Amit, Field Theory, the Renormalization Group, and Critical Phenomena (McGraw-Hill, New York, 1978).

${ }^{13}$ M. Hillery, R. F. O'Connell, M. O. Scully, and E. P. Wigner, Phys. Rep. 106, 121 (1984).

${ }^{14}$ C. Chandler, L. Cohen, C. Lee, M. Scully, and K. Wódkiewicz, Found. Phys. 22, 867 (1992).

${ }^{15}$ E. Fradkin, Field Theories of Condensed Matter Systems (Addison-Wesley, Redwood City, 1991), Chap. 5.

${ }^{16}$ E. Fradkin and M. Stone, Phys. Rev. B 38, 7215 (1988).

${ }^{17}$ A. Perelomov, Generalized Coherent States and Their Applications (Springer, Berlin, 1986).

${ }^{18}$ W. E. Evenson, J. R. Schrieffer, and S. Q. Wang, J. Appl. Phys. 41, 1199 (1970).

${ }^{19}$ S. Leibler and H. Orland, Ann. Phys. (N.Y.) 132, 277 (1981).

${ }^{20}$ For example, T. Moriya, J. Magn. Magn. Mater. 14, 1 (1979).

${ }^{21}$ K. S. Chana, J. H. Samson, M. U. Luchini, and V. Heine, J. Phys. Condens. Matter 3, 6455 (1991).

22J. E. Hirsch, Phys. Rev. B 28, 4059 (1983).

${ }^{23}$ G. Dopf, A. Muramatsu, and W. Hanke, Europhys. Lett. 17, 559 (1992) 
${ }^{24}$ E. Y. Loh, Jr., J. E. Gubernatis, R. T. Scalettar, S. R. White, D. J. Scalapino, and R. L. Sugar, Phys. Rev. B 41, 9301 (1990).

${ }^{25}$ Y. Alhassid, D. J. Dean, S. E. Koonin, G. Lang, and W. E. Ormand, Phys. Rev. Lett. 72, 613 (1994).

26J. H. Samson, Phys. Rev. B 47, 3408 (1993).

27J. H. Samson, in Quantum Monte Carlo Methods in Condensed Matter Physics, edited by M. Suzuki (World Scientific, Singapore, 1993), p. 235.

${ }^{28}$ M. V. Berry, Proc. R. Soc. London, Ser. A 392, 45 (1984).

${ }^{29}$ See, e.g., T. Kawata, Fourier Analysis in Probability Theory
(Academic, New York, 1972), Sec. 10.3.

${ }^{30}$ S. Sorella, S. Baroni, R. Car, and M. Parrinello, Europhys. Lett. 8, 663 (1989).

${ }^{31}$ A. Angelucci and G. Jug, Int. J. Mod. Phys. B 3, 1069 (1989); A. Angelucci, Phys. Rev. B 44, 6849 (1991).

32J. H. Samson, Phys. Rev. B 30, 1437 (1984).

${ }^{33}$ P. Coleman, J. Magn. Magn. Mater. 47-48, 323 (1985).

${ }^{34}$ J. H. Samson, J. Magn. Magn. Mater. 54-57, 983 (1986).

${ }^{35}$ C. Kittel and H. Shore, Phys. Rev. 138, A1165 (1965).

36J. H. Samson, J. Phys. (Paris) 45, 1675 (1984). 